\title{
Clinical use of the diagnostic criteria for major depressive disorder
}

\author{
C. Barbui, Editor*
}

In this Issue of Epidemiology and Psychiatric Sciences two Editorials discuss the epidemiological and clinical implications of the current diagnostic criteria for major depressive disorder (MDD). Considering that the term 'diagnosis' is usually used to indicate an attempt at classification of an individual's condition into separate and distinct categories that allow clinical decisions about prognosis and treatment to be made, under debate is whether MDD diagnostic criteria capture the fundamental nature of this syndrome, and whether making a diagnosis of MDD is a guide for clinical action.

In clinical practice, diagnoses are needed for characterising an individual's condition (what goes wrong and why), for prognostic reasons (expectations for the future) and for treatment implications (how a condition can be improved). Of note, not only health care professionals, but also patients and family members need detailed information about these compelling key aspects.

In the first Editorial, Lorenzo-Luaces (2015) a critique of the MDD diagnostic concept is made from an epidemiological-evidence angle. Based on prevalence data from retrospective studies and prospective epidemiological surveys, Lorenzo-Luaces noted that current MDD criteria tend to lump together depressive disorders with a severe, chronic and recurrent course, often non-responsive to treatment, with depressive disorders that are adaptive sadness reactions or singleepisode afflictions that are mild, unlikely to recur, and often responsive to placebo. On these grounds, Lorenzo-Luaces concluded that the current MDD criteria can be applied to identify an heterogeneous continuum of depressive conditions ranging from normal periods of sadness (the 'common cold') to severe and recurrent depressive episodes ('highly debilitating illness'). This argument, substantiated by a careful critical review of epidemiological and clinical data, including evidence from randomised studies, would suggest that a better (or different) definition of MDD is needed: one that could divide those who are 'truly ill' from those who are not 'truly ill', those with a

* Address for correspondence: Professor C. Barbui, Department of Public Health and Community Medicine, Section of Psychiatry, Ospedale Policlinico 'G.B. Rossi', Piazzale L.A. Scuro 10, 37134 Verona, Italy.

(Email: corrado.barbui@univr.it) mild prognosis from those with a severe course and outcome, those who are responsive to treatment from those who are less likely to respond.

The second Editorial Patten (2015) further expands these notions by pointing out some critical issues. While Lorenzo-Luaces described heterogeneity between individuals fulfilling diagnostic criteria for MDD, Scott Patten, in the second Editorial, suggested that the concept of heterogeneity may similarly be used 'within individuals'. Individuals with a diagnosis of MDD may experience both mild and severe depressive episodes, and therefore the idea of decreasing heterogeneity by using severity as a criterion might not be feasible in practice. Consequently, the problem of differentiating those who are truly ill from those who are not cannot be solved by putting a different threshold to MDD criteria, as inevitably this would just produce more or less restrictive criteria, but the fundamental nature of this syndrome would not be better captured - in other words the heterogeneity of presentation and course are not merely issues of classification but are inherent in the clinical problem itself.

Both Editorialists seem to convey a similar message on the (lack of) usefulness of making a diagnosis of MDD in clinical practice. Current diagnostic criteria do not identify a homogeneous condition, prognosis may be highly variable, and treatment response cannot be predicted. On these grounds, its usefulness in practice may be rather limited. But it happens that in clinical practice clinicians do make this diagnosis very often. Why is it so? Is it because clinicians tend to overestimate its ability to guide the selection of treatments and prediction of prognosis, viewing the diagnosis as being more real than it really is - a concept that Scott Patten calls 'reification', i.e. treating an abstraction as if it were a real thing?

As a practicing doctor I would argue that reification is a concept that brilliantly applies to several different levels of care which may be responsible for the use of MDD diagnostic criteria in clinical practice: (1) at epidemiological level, prevalence and incidence studies are strictly focused on MDD criteria, thus treating this syndromal abstraction as if it were a real thing; (2) both pharmacological and non-pharmacological clinical trials enrol patient populations that strictly meet MDD criteria, as if this abstraction were a real thing; (3) medicine regulatory agencies approve new 
medicines for use in individuals with MDD only, viewing the diagnosis as being more real than it really is; (4) guideline producers recommend psychological interventions for use in individuals with MDD only, viewing the diagnosis as being more real than it really is; (5) for accountability reasons, policy makers collect summary statistics on numbers of people fulfilling MDD diagnostic criteria, as if all individuals with depressive symptoms that we treat every day might be forced into these criteria.

It is this general context that inevitably leads clinicians to regularly use this diagnostic concept. It fulfils more a formal requirement than a clinical need, in particular that of being accountable and that of being coherent by matching clinical decisions, including treatment choices (i.e. use of antidepressants and/or non-pharmacological interventions) with standards of care (medicine labels and guidelines, for example). As a consequence, in clinical practice the flow is not clinical presentation $>$ diagnosis $>$ prognosis $>$ treatment, but clinical presentation (what goes wrong and why) $>$ prognosis (expectations for the future) $>$ treatment (how this condition can be improved) $>$ diagnosis (formal label to justify clinical decisions and treatment choices). I therefore tend to sympathise with Scott Patten's conclusion that '[MDD]... should not be regarded as an effective direct guide for clinical action'.

It seems similarly reasonable that Lorenzo-Luaces concluded his Editorial by calling for more research on bio-psychological factors and contextual variables that may help distinguish, within the heterogeneous group of conditions that we call MDD, more homogeneous subgroups of individuals at similar prognosis, in which clinicians may know in advance which treatment is more likely to be beneficial. This information is still missing.

\section{Acknowledgement}

None.

\section{Financial Support}

None.

\section{Conflict of Interest}

None.

\section{References}

Lorenzo-Luaces L (2015). From the common cold to a highly debilitating and recurrent illness. Epidemiology and Psychiatric Sciences, this issue.

Patten S (2015). Major depressive disorder: reification and (maybe) rheostasis. Epidemiology and Psychiatric Sciences, this issue. 